\title{
POCS-Based Texture Reconstruction Method Using Clustering Scheme by Kernel PCA
}

\author{
Takahiro OGAWA $^{\dagger a}$, Student Member and Miki HASEYAMA ${ }^{\dagger}$, Member $^{\text {Mem }}$
}

\begin{abstract}
SUMMARY A new framework for reconstruction of missing textures in digital images is introduced in this paper. The framework is based on a projection onto convex sets (POCS) algorithm including a novel constraint. In the proposed method, a nonlinear eigenspace of each cluster obtained by classification of known textures within the target image is applied to the constraint. The main advantage of this approach is that the eigenspace can approximate the textures classified into the same cluster in the leastsquares sense. Furthermore, by monitoring the errors converged by the POCS algorithm, a selection of the optimal cluster to reconstruct the target texture including missing intensities can be achieved. This POCS-based approach provides a solution to the problem in traditional methods of not being able to perform the selection of the optimal cluster due to the missing intensities within the target texture. Consequently, all of the missing textures are successfully reconstructed by the selected cluster's eigenspaces which correctly approximate the same kinds of textures. Experimental results show subjective and quantitative improvement of the proposed reconstruction technique over previously reported reconstruction techniques. key words: texture, reconstruction, kernel, PCA, POCS
\end{abstract}

\section{Introduction}

In the field of image restoration, reconstruction of missing areas in digital images is a very important issue because it has a number of fundamental applications. For example, it is applied to removal of unnecessary objects such as superimposed text, restoration of corrupted old films, and interpolation of missing blocks transmitted in an error-prone environment for video communications. Many methods for achieving accurate reconstruction of important image features, such as edges and textures, have been proposed for these applications. Most algorithms reported in the literature are based on structure inpainting techniques for accurate reconstruction of missing edges [1]-[3]. These techniques are effective for pure structure images. However, since ordinary images contain many textures, different techniques work better for those parts. Thus, several texture synthesis methods have also been proposed for accurate reconstruction of missing textures [4]-[5].

In recent work, the study of kernel methods has developed rapidly and its achievements have become a center of attraction [6]. Kernel PCA (principal component analysis) is also suitable for extraction of nonlinear features in observed

Manuscript received December 18, 2006.

Manuscript revised March 16, 2007.

Final manuscript received April 16, 2007.

†The authors are with the Graduate School of Information Science and Technology, Hokkaido University, Sapporo-shi, 0600814 Japan.

a) E-mail: ogawa@1md.ist.hokudai.ac.jp

DOI: 10.1093/ietfec/e90-a.8.1519 data [7]. By utilizing projection schemes onto the nonlinear subspace obtained in the kernel PCA, the missing textures can be reconstructed from the known textures. However, such approaches must be based on the assumption that the squared distances between arbitrary local areas within the target image are small enough. This means that the target image must consist of only one type of texture. Thus, when various kinds of textures exist in the target image, it is desirable that the missing textures be reconstructed from only the same kinds of textures. Unfortunately, these textures cannot be selected by traditional methods since the distance between the target area including the missing intensities and the other areas cannot be calculated. The method proposed in [8] selects sample subimages, and can reconstruct missing textures by using the convex sets defined from the selected subimages. However, since these subimages must be selected manually, fully automatic texture reconstruction cannot be realized.

In this paper, a novel texture reconstruction method based on the theory of projections onto convex sets (POCS) is proposed. The POCS algorithm has been applied to blocking artifact reduction in coded images as a nonlinear image restoration method [9], [10]. In the proposed method, the following two novel approaches have been introduced into the POCS algorithm for achieving accurate restoration of the target image that includes various textures. One approach is the use of a new constraint in the POCS algorithm. Texture classification is performed for known local images, and the nonlinear eigenspace of each cluster is applied to the constraint of the POCS algorithm. The main advantage of this approach is that each nonlinear eigenspace correctly approximates the local images classified into the same cluster. Furthermore, by introducing the other approach into the POCS algorithm, optimal cluster selection for the target local image including missing intensities can be performed. Special attention is given to the errors converged by the POCS algorithm. These values correspond to the distances from the eigenspace of each cluster, and the optimal cluster can therefore be selected automatically even if the target local image includes missing intensities. This is the biggest advantage of the proposed method. Therefore, the POCS algorithm, whose constraint utilizes the selected cluster's eigenspace, can successfully reconstruct the target local image. Consequently, adaptive restoration of the missing textures can be achieved by using the proposed method, and the performance of the proposed method is better than the performances of traditional methods. Further, the pro- 


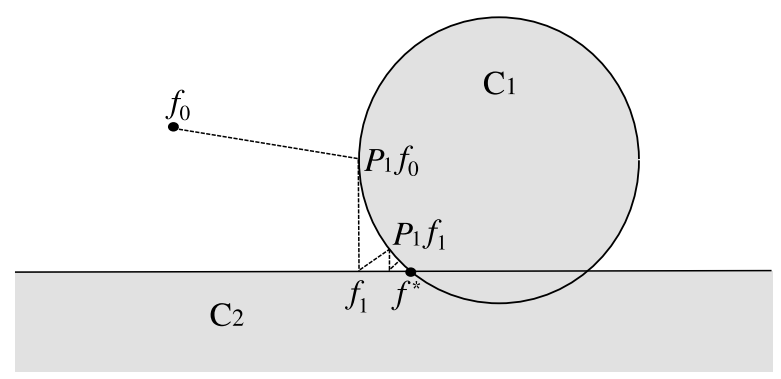

Fig. 1 An illustration of the recursion in Eq. (2) at work.

posed method realizes the fully automatic reconstruction.

This paper is organized as follows. The POCS algorithm is explained in Sect. 2. In Sect. 3, a novel texture reconstruction method using the POCS algorithm is presented. Experimental results that verify the good performance of the proposed method are presented in Sect. 4.

\section{POCS Algorithm}

The POCS algorithm is explained in this section. The theory of POCS was first introduced to the field of image restoration by Youla and Webb. This algorithm estimates the original image $f$ in the Hilbert space $H$ from its known properties. Given $n$ properties of the original image $f$, these properties generate $n$ well-defined closed convex sets $C_{i}$ $(i=1,2, \cdots, n)$. The original image $f$ should be included in all of the $C_{i}$ 's and also the intersection of all of the $C_{i}$ 's $C^{*}$, i.e.,

$$
f \in C^{*}=\bigcap_{i=1}^{n} C_{i}
$$

It is clear that the intersection $C^{*}$, which includes $f^{*}$, is a closed and convex set. Consequently, regardless of whether $C^{*}$ includes elements other than $f^{*}$, the problem of estimating $f$ from its $n$ properties is equivalent to finding at least one point $f^{*}$ belonging to $C^{*}$. Unfortunately, $C^{*}$ may be nonlinear and complex in structure, making a direct estimation of $f^{*}$ is almost impossible. However, as shown in Fig. 1, given the projection operator $P_{i}$ onto $C_{i}$, the iteration

$$
f_{t}=P_{n} P_{n-1} \cdots P_{2} P_{1} f_{t-1} \quad t=1,2, \cdots
$$

converges to a limiting point $f^{*}$ of the intersection $C^{*}$ for an arbitrary initial element $f_{0}$ in $H$. Note that the operator $P_{i}$ satisfies

$$
\left\|f-P_{i} f\right\|=\min _{g \in C_{i}}\|f-g\|,
$$

where $\|\cdot\|$ denotes the norm in $H$. Then we can calculate $f^{*}$ from the $n$ properties of the original image by using Eq. (2).

\section{Our Reconstruction Algorithm}

A POCS-based texture reconstruction method is presented in this section. In order to successfully reconstruct a target image containing several textures, it is necessary to know

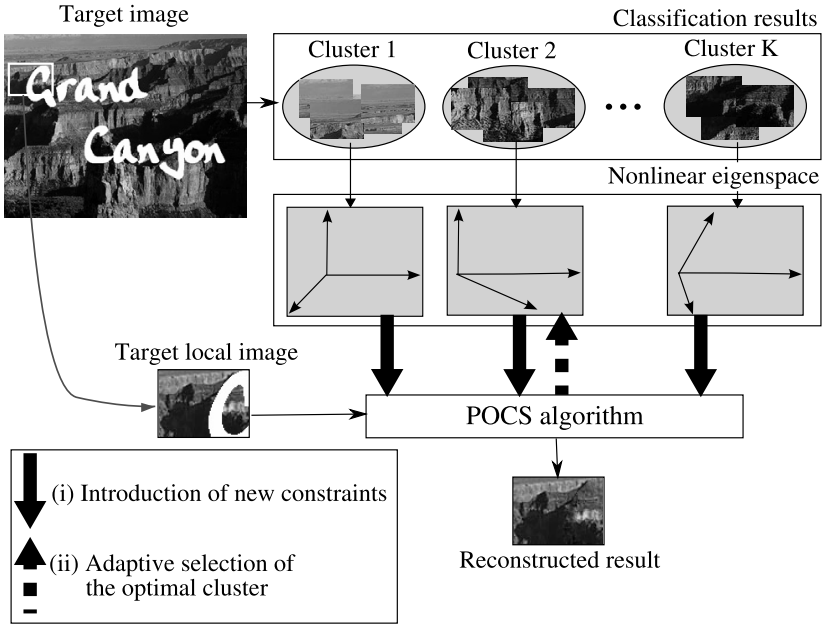

Fig. 2 Outline of the proposed POCS-based texture reconstruction algorithm.

which parts have the same kinds of textures. Therefore, a texture classification is performed for local images within the target image before restoring the missing textures.

Figure 2 shows an outline of our texture reconstruction method. From the classification results, a target local image including missing textures is reconstructed by using the POCS algorithm. In this procedure, the following two novel approaches are introduced into the POCS algorithm:

(i) Introduction of the nonlinear eigenspace of each cluster obtained by the texture classification into the constraint of the POCS algorithm.

(ii) Adaptive selection of the optimal cluster for the target local image based on errors caused by the POCS algorithm.

The first approach aims at accurate texture approximation of the local images included in the same clusters. Further, the second approach is necessary to restore the target local image from only the images containing the same kinds of textures. By utilizing the above approaches, adaptive reconstruction of the missing textures can be achieved, so that successful reconstruction of the target image including various kinds of textures can be expected.

In order to perform the two approaches, we need to classify local images that do not include missing textures in advance. The classification procedure is described in detail in 3.1 , and the reconstruction algorithm of the missing textures is presented in 3.2.

\subsection{Texture Classification Algorithm}

In this subsection, classification of textures within the target image into $K$ clusters as preprocessing for reconstruction of the missing textures is described. First, we clip $N$ local images $f_{i}(w \times h$ pixels, $i=1,2, \cdots, N)$ not including missing textures from the target image and generate vectors $\mathbf{x}_{i} \in \mathbf{R}^{w h}$, whose elements are the raster scanned intensities of $f_{i}$. Then, we map $\mathbf{x}_{i}$ into the feature space $\mathcal{F}$ via the 
nonlinear map [6] and obtain $\phi\left(\mathbf{x}_{i}\right)$. Note that the mapped results $\phi\left(\mathbf{x}_{i}\right)$ are high-dimensional or infinite-dimensional, and it therefore may not be possible to calculate them directly. Fortunately, it is well known that the following computational procedures depend only on the inner products in the feature space, which can be efficiently obtained from a suitable kernel function $k(\cdot, \cdot)$ [11]. Specifically, given arbitrary vectors $\mathbf{x}, \mathbf{y}\left(\in \mathbf{R}^{w h}\right)$, the inner product $\phi(\mathbf{x})^{\prime} \phi(\mathbf{y})$ is calculated as follows:

$$
\phi(\mathbf{x})^{\prime} \phi(\mathbf{y})=\kappa(\mathbf{x}, \mathbf{y})
$$

where vector/matrix transpose (in both the input and feature spaces) is denoted by the superscript ' in this paper. In Eq. (4), several functions (e.g., Gaussian, polynomial, or sigmoid ) can be applied to $\kappa(\cdot, \cdot)$.

The proposed method regards the mapped results $\phi\left(\mathbf{x}_{i}\right)$ $(i=1,2, \cdots, N)$ as texture feature vectors and performs their classification that minimizes the following new criterion ${ }^{\dagger}$ :

$$
F=\sum_{k=1}^{K} \sum_{j=1}^{M^{k}}\left\|\phi_{j}^{k}-\mathbf{U}^{k} \mathbf{U}^{k^{\prime}} \phi_{j}^{k}\right\|^{2}
$$

where $\phi_{j}^{k}\left(j=1,2, \cdots, M^{k}\right)$ is $\phi\left(\mathbf{x}_{i}\right)(i=1,2, \cdots, N)$ belonging to cluster $k$, and $M^{k}$ is the number of $\phi\left(\mathbf{x}_{i}\right)$ belonging to cluster $k$. Furthermore, given $\boldsymbol{\Xi}^{k}=\left[\phi_{1}^{k}, \phi_{2}^{k}, \cdots, \phi_{M^{k}}^{k}\right]$,

$$
\mathbf{U}^{k}=\left[\mathbf{u}_{1}^{k}, \mathbf{u}_{2}^{k}, \cdots, \mathbf{u}_{D^{k}}^{k}\right] \quad\left(D^{k}<M^{k}\right)
$$

is an eigenvector matrix of $\boldsymbol{\Xi}^{k} \mathbf{H}^{k} \mathbf{H}^{k} \boldsymbol{\Xi}^{k^{\prime}}$ and $\mathbf{U}^{k} \mathbf{U}^{k^{\prime}}$ is the projection matrix onto the eigenspace spanned by their eigenvectors $\mathbf{u}_{d}^{k}\left(d=1,2, \cdots, D^{k}\right)$. The matrix $\mathbf{H}^{k}$ is a centering matrix and necessary for calculating the covariance matrix $\boldsymbol{\Xi}^{k} \mathbf{H}^{k} \mathbf{H}^{k} \boldsymbol{\Xi}^{k^{\prime}}$ to obtain the eigenvector matrix $\mathbf{U}^{k}$. It is defined as follows:

$$
\mathbf{H}^{k}=\mathbf{I}-\frac{1}{M^{k}} \mathbf{1 1}^{\prime},
$$

where $\mathbf{I}$ is the $M^{k} \times M^{k}$ identity matrix and $\mathbf{1}=[1,1, \cdots, 1]^{\prime}$ is an $M^{k} \times 1$ vector. In Eq. (6), $D^{k}$ is the dimension of the eigenspace of cluster $k$, and it is set to the value whose cumulative proportion $\mathrm{cp}^{k}$ is larger than $T h$. The cumulative proportion $\mathrm{cp}^{k}$ is calculated as follows:

$$
\mathrm{cp}^{k}=\frac{\sum_{d=1}^{D^{k}} \lambda_{d}^{k}}{\sum_{d=1}^{M^{k}} \lambda_{d}^{k}},
$$

where $\lambda_{d}^{k}\left(\lambda_{1}^{k} \geq \lambda_{2}^{k} \geq \cdots \geq \lambda_{M^{k}}^{k}\right)$ is the eigenvalue of the eigenvector $\mathbf{u}_{d}^{k}$.

In the proposed method, the nonlinear eigenspace of $\boldsymbol{\Xi}^{k} \mathbf{H}^{k} \mathbf{H}^{k} \boldsymbol{\Xi}^{k^{\prime}}$ correctly approximates $\phi_{j}^{k}\left(j=1,2, \cdots, M^{k}\right)$ belonging to the same cluster $k$ in the least-squares sense. Therefore, from all subspaces with the dimension $D^{k}$, we utilize the eigenspace of $\Xi^{k} \mathbf{H}^{k} \mathbf{H}^{k} \boldsymbol{\Xi}^{k^{\prime}}$. Then, it leads to satisfying texture reconstruction results.

Next, we explain the reason why we utilize the kernel method for calculating the eigenspace. In contrast to linear eigenspace, nonlinear eigenspace is capable of capturing part of the higher-order statistics which are particularly important for encoding image structure [13]. According to this characteristic, we utilize the kernel method in order to accurately approximate textures in the eigenspace with the dimension $D^{k}$ which is much lower than $w h$.

In Eq. (6), the eigenvectors $\mathbf{u}_{d}^{k}\left(d=1,2, \cdots, D^{k}\right)$ are high-dimensional, and Eq. (5) therefore cannot be calculated directly. Thus, we introduce the computational scheme shown in Eq. (4) into the calculation of Eq. (5). The eigenvector matrix $\mathbf{U}^{k}$ satisfies the following singular value decomposition:

$$
\boldsymbol{\Xi}^{k} \mathbf{H}^{k} \cong \mathbf{U}^{k} \boldsymbol{\Lambda}^{k} \mathbf{V}^{k^{\prime}},
$$

where $\boldsymbol{\Lambda}^{k}$ is the eigenvalue matrix and $\mathbf{V}^{k}$ is the eigenvector matrix of $\mathbf{H}^{k} \Xi^{k^{\prime}} \boldsymbol{\Xi}^{k} \mathbf{H}^{k}$. Therefore, $\mathbf{U}^{k}$ can be obtained as follows:

$$
\mathbf{U}^{k} \cong \boldsymbol{\Xi}^{k} \mathbf{H}^{k} \mathbf{V}^{k} \boldsymbol{\Lambda}^{k^{-1}}
$$

Then Eq. (5) can be rewritten as

$$
\begin{aligned}
F \cong & \sum_{k=1}^{K} \sum_{j=1}^{M^{k}}\left\|\phi_{j}^{k}-\boldsymbol{\Xi}^{k} \mathbf{H}^{k} \mathbf{V}^{k} \boldsymbol{\Lambda}^{k^{-2}} \mathbf{V}^{k^{\prime}} \mathbf{H}^{k} \boldsymbol{\Xi}^{k^{\prime}} \phi_{j}^{k}\right\|^{2} \\
= & \sum_{k=1}^{K} \sum_{j=1}^{M^{k}}\left\|\phi_{j}^{k}-\boldsymbol{\Xi}^{k} \mathbf{T}^{k} \boldsymbol{\Xi}^{k^{\prime}} \phi_{j}^{k}\right\|^{2} \\
= & \sum_{k=1}^{K} \sum_{j=1}^{M^{k}}\left\{\phi_{j}^{k^{\prime}} \phi_{j}^{k}+\phi_{j}^{k^{\prime}} \boldsymbol{\Xi}^{k} \mathbf{T}^{k^{\prime}} \boldsymbol{\Xi}^{k^{\prime}} \boldsymbol{\Xi}^{k} \mathbf{T}^{k} \Xi^{k^{\prime}} \phi_{j}^{k}\right. \\
& \left.-2 \phi_{j}^{k^{\prime}} \boldsymbol{\Xi}^{k} \mathbf{T}^{k} \boldsymbol{\Xi}^{k^{\prime}} \phi_{j}^{k}\right\} \\
= & \sum_{k=1}^{K} \sum_{j=1}^{M^{k}}\left\{\kappa\left(\mathbf{x}_{j}^{k}, \mathbf{x}_{j}^{k}\right)\right. \\
& +\kappa\left(\mathbf{X}^{k}, \mathbf{x}_{j}^{k}\right)^{\prime} \mathbf{T}^{k^{\prime}} \boldsymbol{\kappa}\left(\mathbf{X}^{k}, \mathbf{X}^{k}\right) \mathbf{T}^{k} \kappa\left(\mathbf{X}^{k}, \mathbf{x}_{j}^{k}\right) \\
& \left.-2 \kappa\left(\mathbf{X}^{k}, \mathbf{x}_{j}^{k}\right)^{\prime} \mathbf{T}^{k} \boldsymbol{\kappa}\left(\mathbf{X}^{k}, \mathbf{x}_{j}^{k}\right)\right\},
\end{aligned}
$$

where

$$
\begin{aligned}
\mathbf{T}^{k} & =\mathbf{H}^{k} \mathbf{V}^{k} \boldsymbol{\Lambda}^{k^{-2}} \mathbf{V}^{k^{\prime}} \mathbf{H}^{k}, \\
\mathbf{X}^{k} & =\left[\mathbf{x}_{1}^{k}, \mathbf{x}_{2}^{k}, \cdots, \mathbf{x}_{M^{k}}^{k},\right.
\end{aligned}
$$

and $\mathbf{x}_{j}^{k}\left(j=1,2, \cdots, M^{k}\right)$ are the vectors $\mathbf{x}_{i}(i=1,2, \cdots, N)$ belonging to cluster $k$. Furthermore, $\kappa\left(\mathbf{X}^{k}, \mathbf{x}_{j}^{k}\right)$ and $\kappa\left(\mathbf{X}^{k}, \mathbf{X}^{k}\right)$ are defined as follows:

$$
\begin{aligned}
& \kappa\left(\mathbf{X}^{k}, \mathbf{x}_{j}^{k}\right)= {\left[\kappa\left(\mathbf{x}_{1}^{k}, \mathbf{x}_{j}^{k}\right), \kappa\left(\mathbf{x}_{2}^{k}, \mathbf{x}_{j}^{k}\right), \cdots, \kappa\left(\mathbf{x}_{M^{k}}^{k}, \mathbf{x}_{j}^{k}\right)\right]^{\prime}, } \\
& \kappa\left(\mathbf{X}^{k}, \mathbf{X}^{k}\right)=\left[\begin{array}{cccc}
\kappa\left(\mathbf{x}_{1}^{k}, \mathbf{x}_{1}^{k}\right) & \kappa\left(\mathbf{x}_{1}^{k}, \mathbf{x}_{2}^{k}\right) & \ldots & \kappa\left(\mathbf{x}_{1}^{k}, \mathbf{x}_{M^{k}}^{k}\right) \\
\kappa\left(\mathbf{x}_{2}^{k}, \mathbf{x}_{1}^{k}\right) & \kappa\left(\mathbf{x}_{2}^{k}, \mathbf{x}_{2}^{k}\right) & \ldots & \kappa\left(\mathbf{x}_{2}^{k}, \mathbf{x}_{M^{k}}^{k}\right) \\
\vdots & \vdots & \ddots & \vdots \\
\kappa\left(\mathbf{x}_{M^{k}}^{k}, \mathbf{x}_{1}^{k}\right) & \kappa\left(\mathbf{x}_{M^{k}}^{k}, \mathbf{x}_{2}^{k}\right) & \ldots & \kappa\left(\mathbf{x}_{M^{k}}^{k}, \mathbf{x}_{M^{k}}^{k}\right.
\end{array}\right] .
\end{aligned}
$$

In Eq. (5), the mean of $\phi_{j}^{k}\left(j=1,2, \cdots, M^{k}\right)$ must be subtracted from $\phi_{j}^{k}$ before projecting onto the nonlinear eigenspace, and it must be added to the projected result. The same procedures are necessary for Eq. (15) in the following subsection. In this paper, we do not show these procedures for brief explanation. The details of these procedures are shown in [12]. 


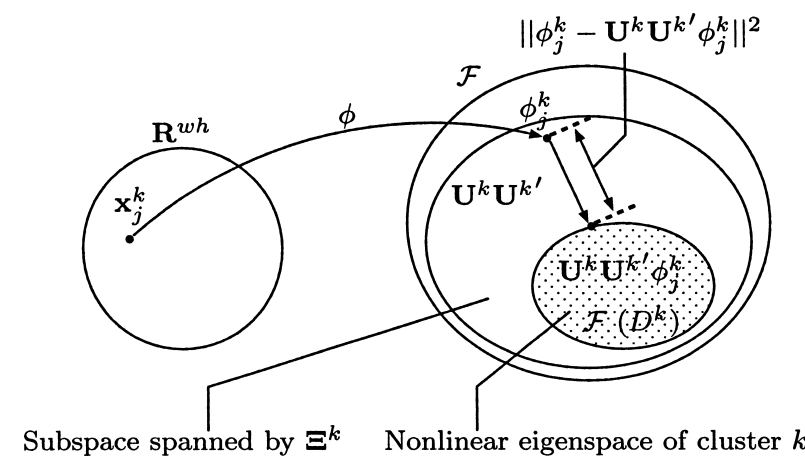

Fig. 3 Relationship between $\phi_{j}^{k}$ and $\mathbf{U}^{k} \mathbf{U}^{k^{\prime}} \phi_{j}^{k}$. The squared error $\| \phi_{j}^{k}-$ $\mathbf{U}^{k} \mathbf{U}^{k^{\prime}} \phi_{j}^{k} \|^{2}$ corresponds to the distance between $\phi_{j}^{k}$ and the eigenspace of cluster $k$.

In Eq. (5), $\mathbf{U}^{k} \mathbf{U}^{k^{\prime}}$ is the projection matrix onto the eigenspace spanned by their eigenvectors $\mathbf{u}_{d}^{k}(d=$ $\left.1,2, \cdots, D^{k}\right)$. Therefore, as shown in Fig. 3 , criterion $F$ represents the sum of the approximation errors of $\phi_{j}^{k}(j=$ $\left.1,2, \cdots, M^{k}\right)$ in their eigenspaces. This means that the squared error in Eq. (5) corresponds to the distance from the nonlinear eigenspace of each cluster. Furthermore, since we regard $\phi\left(\mathbf{x}_{i}\right)(i=1,2, \cdots, N)$ as the texture feature vectors of $\mathbf{x}_{i}$, the new criterion $F$ is useful for classification of the textures.

In the texture classification using Eq. (11), we must assign all $\phi\left(\mathbf{x}_{i}\right)(i=1,2, \cdots, N)$ to $K$ clusters and select one result that minimizes the criterion $F$. Unfortunately, the combination is ${ }_{N} C_{M^{1}}{ }_{N-M^{1}} C_{M^{2}} \cdots,{ }_{M^{K}} C_{M^{K}}$, and searching for the optimal solution from all of the classification results therefore involves a high computational cost. Thus, we first set the dimension of each eigenspace to one and iterate the classification procedure which assigns $\phi\left(\mathbf{x}_{i}\right)$ to the cluster minimizing

$$
\begin{aligned}
\tilde{F}_{i}^{k}(t)= & \left\|\phi\left(\mathbf{x}_{i}\right)-\mathbf{U}_{t}^{k} \mathbf{U}_{t}^{k^{\prime}} \phi\left(\mathbf{x}_{i}\right)\right\|^{2} \\
\cong & \kappa\left(\mathbf{x}_{i}, \mathbf{x}_{i}\right) \\
& +\kappa\left(\mathbf{X}_{t}^{k}, \mathbf{x}_{i}\right)^{\prime} \mathbf{T}_{t}^{k^{\prime}} \kappa\left(\mathbf{X}_{t}^{k}, \mathbf{X}_{t}^{k}\right) \mathbf{T}_{t}^{k} \kappa\left(\mathbf{X}_{t}^{k}, \mathbf{x}_{i}\right) \\
& -2 \kappa\left(\mathbf{X}_{t}^{k}, \mathbf{x}_{i}\right)^{\prime} \mathbf{T}_{t}^{k} \kappa\left(\mathbf{X}_{t}^{k}, \mathbf{x}_{i}\right) .
\end{aligned}
$$

where $\mathbf{U}_{t}^{k}, \mathbf{X}_{t}^{k}$, and $\mathbf{T}_{t}^{k}$ respectively represent $\mathbf{U}^{k}, \mathbf{X}^{k}$, and $\mathbf{T}^{k}$ of the $t$-th iteration.

Here, we explain how to determine $M^{k}$. First, the maximum number of $M^{k}$ is denoted as $M_{\max }$ and it is computed as follows: If $\frac{N}{K}$ is an integer, $M_{\max }=\frac{N}{K}$. Otherwise, $M_{\max }=\left\lceil\frac{N}{K}\right\rceil$. Further, in order to obtain the value $M^{k}$, we firstly set the initial value $M^{k}=0$ and repeat the following procedures until all of $\phi\left(\mathbf{x}_{i}\right)$ are assigned to $K$ clusters.

1) The numbers $k$ and $i$, which minimize $\tilde{F}_{i}^{k}(t)$, are calculated and denoted by $k_{\min }$ and $i_{\min }$, respectively.

2) If the number of elements assigned to cluster $k_{\min }$ is less than $M_{\max }$ and $\phi\left(\mathbf{x}_{i_{\min }}\right)$ is not assigned to any clusters, $\phi\left(\mathbf{x}_{i_{\min }}\right)$ is assigned to cluster $k_{\min }$, and $M^{k_{\min }}$ is increased by one.

3) The maximum value of $\tilde{F}_{i}^{k}(t)$, which is denoted as

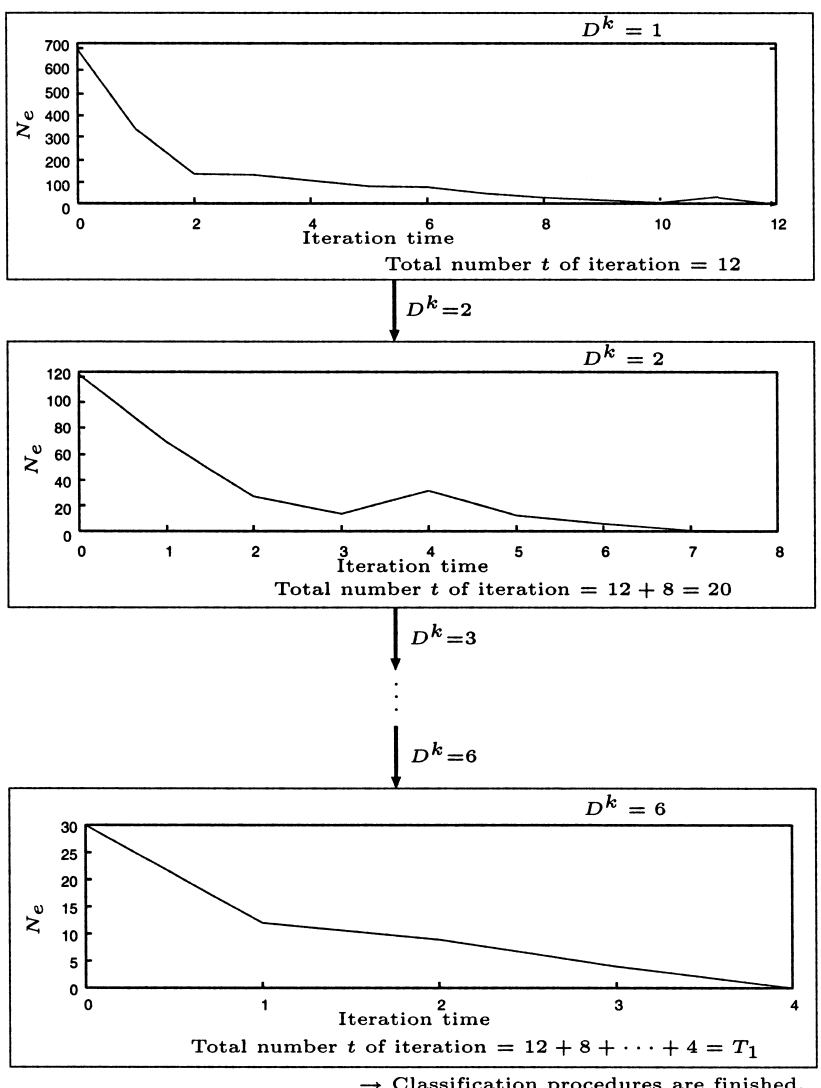

Fig. 4 Example of texture classification in the proposed method. The value $N_{e}$ represents the number of $\phi\left(\mathbf{x}_{i}\right)$ which are assigned to different clusters.

$$
\tilde{F}_{i_{\max }}^{k_{\max }}(t) \text {, is substituted into } \tilde{F}_{i_{\min }}^{k_{\min }}(t) \text {. }
$$

Finally, $M^{k}$ of cluster $k$ is automatically determined.

Further, if any $\phi\left(\mathbf{x}_{i}\right)$ are not assigned to different clusters in the iteration, we increase the dimension of each cluster's eigenspace by one and iterate this classification procedure as shown in Fig. 4. Then, the classification result obtained after $T_{1}$ iteration (i.e., $t=T_{1}$ ) becomes the final texture clustering result. Note that we have to know $\mathbf{U}_{1}^{k}$ $(k=1,2, \cdots, K)$ in the first iteration, i.e., the initial onedimensional eigenspace must be calculated before the texture classification procedure using Eq. (14). Thus, the proposed method determines it as follows. First, by using the k-means algorithm [14], $\phi\left(\mathbf{x}_{i}\right)(i=1,2, \cdots, N)$ are classified into $K$ clusters, and $\boldsymbol{\Xi}^{k}(k=1,2, \cdots, K)$ is calculated for each cluster $k$. Further, the one-dimensional eigenspace spanned by $\mathbf{u}_{1}^{k}$, which is the eigenvector of $\boldsymbol{\Xi}^{k} \mathbf{H}^{k} \mathbf{H}^{k} \boldsymbol{\Xi}^{k^{\prime}}$ with the highest eigenvalue, is calculated.

\subsection{Texture Reconstruction Algorithm}

In this subsection, we show reconstruction of the missing textures in the target image by the POCS algorithm from the classification results presented in the previous subsection. From the target image, we clip a local image $f_{0}(w \times h$ pixels $)$ including missing textures and generate a vector $\mathbf{x}$ whose el- 
ements are its raster scanned intensities. For $\mathbf{x}$, the proposed method calculates its reconstruction result $\hat{\mathbf{x}}$, which satisfies the following novel constraints.

\section{[Constraint 1]}

In vector $\hat{\mathbf{x}}$, the known original intensities are fixed.

\section{[Constraint 2]}

In the feature space, the target vector is in the eigenspace spanned by its eigenvectors $\mathbf{u}_{1}^{k}, \mathbf{u}_{2}^{k}, \cdots$, and $\mathbf{u}_{D^{k}}^{k}$ of cluster $k$. Therefore, given the target vector $\phi_{\tilde{\mathbf{x}}}$, it satisfies

$$
\phi_{\tilde{\mathbf{x}}}=\mathbf{U}^{k} \mathbf{U}^{k^{\prime}} \phi_{\tilde{\mathbf{x}}} .
$$

Note that in the proposed method, the final restoration result $\hat{\mathbf{x}}$ is the vector in the input space. Since an exact pre-image, which is the inverse mapping from the feature space back to the input space, typically does not exist [12], $\hat{\mathbf{x}}$ satisfies the following equation:

$$
\hat{\mathbf{x}} \cong \phi^{-1}\left(\mathbf{U}^{k} \mathbf{U}^{k^{\prime}} \phi(\hat{\mathbf{x}})\right) .
$$

Furthermore, the proposed method newly defines a linear map $\mathbf{A}^{k}$ and calculates the approximate solution $\phi^{-1}$ as follows:

$$
\hat{\mathbf{x}} \cong \mathbf{A}^{k} \mathbf{U}^{k} \mathbf{U}^{k^{\prime}} \phi(\hat{\mathbf{x}}),
$$

where $\mathbf{A}^{k}$ is calculated from the following equation:

$$
\mathbf{A}^{k} \boldsymbol{\Xi}^{k} \mathbf{H}^{k}=\mathbf{X}^{k} \mathbf{H}^{k} .
$$

We substitute Eq. (9) into the above equation, and obtain the following equation:

$$
\mathbf{A}^{k} \mathbf{U}^{k} \boldsymbol{\Lambda}^{k} \mathbf{V}^{k^{\prime}} \cong \mathbf{X}^{k} \mathbf{H}^{k}
$$

Then, $\mathbf{A}^{k}$ can be approximately derived as follows:

$$
\mathbf{A}^{k} \cong \mathbf{X}^{k} \mathbf{H}^{k} \mathbf{V}^{k} \mathbf{\Lambda}^{k^{-1}} \mathbf{U}^{k^{\prime}} .
$$

Thus, Eq. (17) can be rewritten as

$$
\begin{aligned}
\hat{\mathbf{x}} & \cong \mathbf{X}^{k} \mathbf{H}^{k} \mathbf{V}^{k} \boldsymbol{\Lambda}^{k^{-1}} \mathbf{U}^{k^{\prime}} \phi(\hat{\mathbf{x}}) \\
& \cong \mathbf{X}^{k} \mathbf{H}^{k} \mathbf{V}^{k} \boldsymbol{\Lambda}^{k^{-2}} \mathbf{V}^{k^{\prime}} \mathbf{H} \Xi^{k^{\prime}} \phi(\hat{\mathbf{x}}) \\
& =\mathbf{X}^{k} \mathbf{T}^{k} \boldsymbol{\kappa}\left(\mathbf{X}^{k}, \hat{\mathbf{x}}\right) .
\end{aligned}
$$

Note that since $\mathbf{A}^{k}$ is approximately calculated in Eq. (20), $\mathbf{x}=\mathbf{A}^{k} \phi(\mathbf{x})$ is not guaranteed for arbitrary vectors $\mathbf{x}$.

Utilizing the POCS algorithm, the proposed method calculates the vector $\hat{\mathbf{x}}$ that satisfies the above two constraints from the initial vector $\mathbf{x}$. Specifically, we respectively utilize these constraints as the closed convex sets $C_{1}, C_{2}$ in Eq. (1) and calculate $\hat{\mathbf{x}}$ by their projection operators $P_{1}, P_{2}$ in Eq. (2), where the number of the iteration is set to $T_{2}$. Note that if the intersection of the two convex sets does not exist, we cannot obtain the result $\hat{\mathbf{x}}$ satisfying the two constraints as shown in Fig. 5. Therefore, the result by the proposed POCS algorithm is different from that shown in Sect. 2. In such a case, the proposed method outputs the result which satisfies [Constraint 1] as $\hat{\mathbf{x}}$. Then, the distance

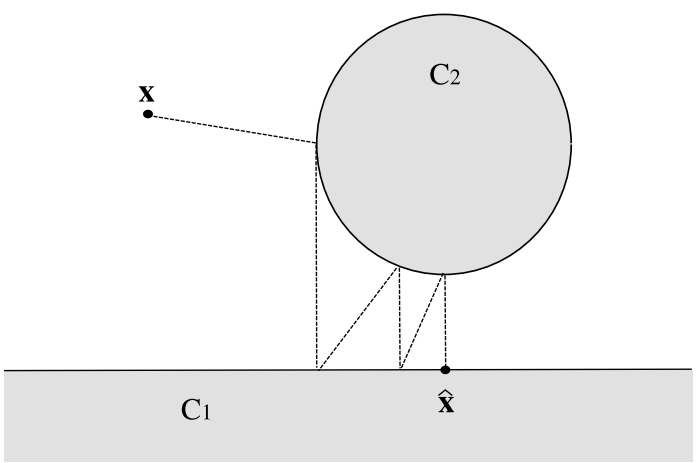

Fig.5 Example of the converged result $\hat{\mathbf{x}}$ by the proposed POCS algorithm.

between the obtained result $\hat{\mathbf{x}}$ and the convex set defined by [Constraint 2] becomes the error of the proposed POCS algorithm.

Given sample data, it is well known their eigenspace minimizes information loss since it is calculated based on the maximum variance criterion. In the least-squares sense, the nonlinear eigenspace used in [Constraint 2] correctly approximates $\phi_{j}^{k}\left(j=1,2, \cdots, M^{k}\right)$ belonging to the same cluster $k$. Therefore, if we can classify $\phi(\mathbf{x})$ of the target local image $f_{0}$, the proposed method accurately reconstructs it by using the eigenspace of the cluster including $\phi(\mathbf{x})$. However, since $\mathbf{x}$ contains missing intensities, $\phi(\mathbf{x})$ cannot be classified by the algorithm shown in 3.1. Thus, in order to achieve the classification of $\mathbf{x}$, the proposed method utilizes the following novel criterion as a substitute for Eq. (14).

$$
\begin{aligned}
E^{k}= & \frac{1}{\operatorname{diag}(\bar{\Sigma})}\left\|\phi(\bar{\Sigma} \hat{\mathbf{x}})-\phi\left(\bar{\Sigma} \mathbf{X}^{k} \mathbf{T}^{k} \kappa\left(\mathbf{X}^{k}, \hat{\mathbf{x}}\right)\right)\right\|^{2} \\
= & \frac{1}{\operatorname{diag}(\bar{\Sigma})}\left\{\phi(\bar{\Sigma} \hat{\mathbf{x}})^{\prime} \phi(\bar{\Sigma} \hat{\mathbf{x}})\right. \\
& +\phi\left(\bar{\Sigma} \mathbf{X}^{k} \mathbf{T}^{k} \kappa\left(\mathbf{X}^{k}, \hat{\mathbf{x}}\right)\right)^{\prime} \phi\left(\bar{\Sigma} \mathbf{X}^{k} \mathbf{T}^{k} \kappa\left(\mathbf{X}^{k}, \hat{\mathbf{x}}\right)\right) \\
& \left.-2 \phi(\bar{\Sigma} \hat{\mathbf{x}})^{\prime} \phi\left(\bar{\Sigma} \mathbf{X}^{k} \mathbf{T}^{k} \kappa\left(\mathbf{X}^{k}, \hat{\mathbf{x}}\right)\right)\right\} \\
= & \frac{1}{\operatorname{diag}(\bar{\Sigma})}\{\kappa(\bar{\Sigma} \hat{\mathbf{x}}, \bar{\Sigma} \hat{\mathbf{x}}) \\
& +\kappa\left(\bar{\Sigma} \mathbf{X}^{k} \mathbf{T}^{k} \kappa\left(\mathbf{X}^{k}, \hat{\mathbf{x}}\right), \bar{\Sigma} \mathbf{X}^{k} \mathbf{T}^{k} \kappa\left(\mathbf{X}^{k}, \hat{\mathbf{x}}\right)\right) \\
& \left.-2 \kappa\left(\bar{\Sigma} \hat{\mathbf{x}}, \bar{\Sigma} \mathbf{X}^{k} \mathbf{T}^{k} \kappa\left(\mathbf{X}^{k}, \hat{\mathbf{x}}\right)\right)\right\},
\end{aligned}
$$

where $\overline{\boldsymbol{\Sigma}}$ is a diagonal matrix whose diagonal elements are zero or one and satisfies $\overline{\mathbf{\Sigma}} \mathbf{x}=\overline{\mathbf{\Sigma}} \hat{\mathbf{x}}$. The criterion $E^{k}$ corresponds to the squared error converged by the POCS algorithm in the feature space. Therefore, this criterion $E^{k}$ as well as $\tilde{F}_{i}^{k}(t)$ in Eq. (14) is applicable for classification of the textures. Selection of the optimal cluster for the target local image including missing intensities then becomes possible. Furthermore, the proposed method regards the result $\hat{\mathbf{x}}$ obtained by the eigenspace of the selected cluster as the output. Consequently, by performing the non-conventional approach, which adaptively selects the optimal eigenspace for the missing texture, we can restore all of the missing textures in the target image accurately. 
As described above, we can reconstruct the missing texture in the target local image. The proposed method clips local images ( $w \times h$ pixels) including missing textures at the same interval (width $\tilde{w}$ pixels, height $\tilde{h}$ pixels) in a raster scanning order and reconstructs them by using the POCS algorithm. Note that each restored pixel has multiple estimation results if the clipping interval is smaller than the size of the local images. In this case, the proposed method regards the result minimizing Eq. (22) as the final one.

\section{Experimental Results}

The performance of the proposed method is shown in this section. Figure $6(\mathrm{a})$ is a test texture image $(480 \times 359$ pixels, 24-bit color levels) that includes the text "Grand Canyon." Figure 6(b) shows the results of reconstruction by the proposed method. In this simulation, we set the parameters of the proposed method as follows: $w=40, h=40, K=8$, $T h=0.9, T_{1}=20, T_{2}=5, \tilde{w}=10$, and $\tilde{h}=10$. Further, the proposed method utilizes the Gaussian kernel for $\kappa(\cdot, \cdot)$ in Eq. (4). The Gaussian kernel is a default "general purpose kernel" in the kernel methods community [13]. It is well known that the nonlinear eigenspace using the Gaussian kernel has higher cumulative proportion than those using the other kernels. In our problem, it can be also verified by Fig. 7. Therefore, in this experiment, we utilize the Gaussian kernel. For comparison, Fig. 6(c) shows the results obtained by the traditional eigenspace method using projection of the nonlinear subspace obtained by the kernel principal component analysis in [6]. We also utilize the
Gaussian kernel in this method. For better subjective evaluation, the enlarged portions around the middle of the images are shown in Figs. 6(d)-(f). It can be seen that the use of the proposed method has achieved noticeable improvements. In the conventional method, different kinds of textures affect the reconstruction of the target missing textures. On the other hand, by selecting the optimal cluster including the same kinds of textures, the proposed method can adaptively reconstruct the missing textures from only the reliable ones. Therefore, the proposed method has higher performance than that of the conventional method. Different

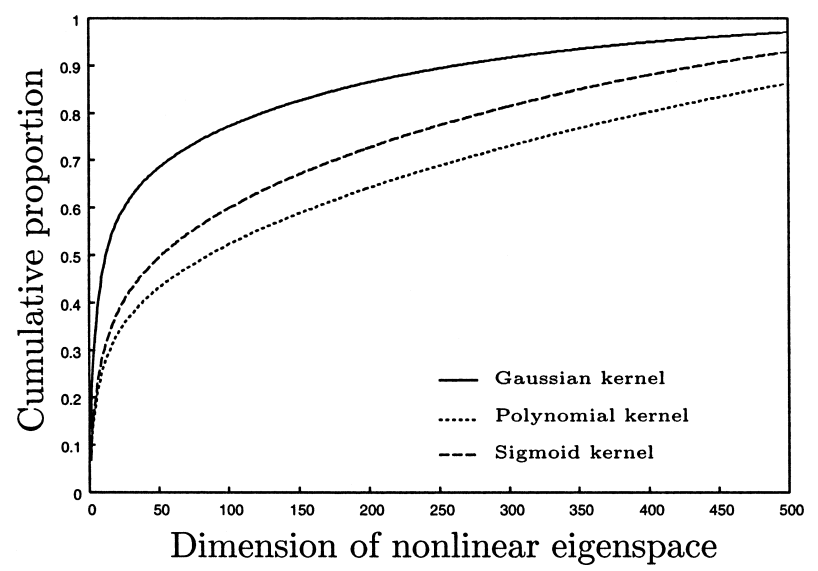

Fig. 7 Relationship between the dimension of the nonlinear eigenspace and its cumulative proportion calculated for the test image shown in Fig. 6(a).

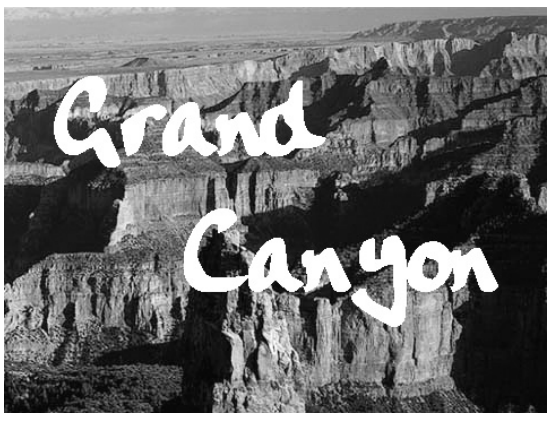

(a)

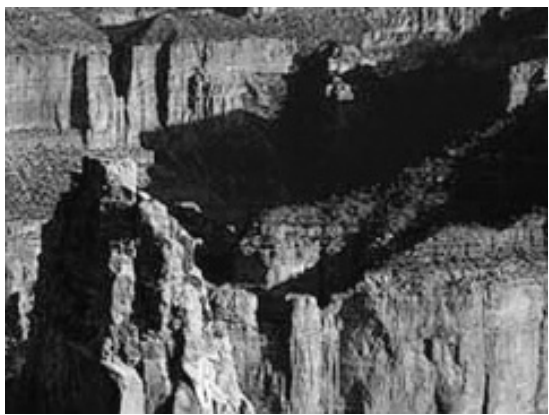

(d)

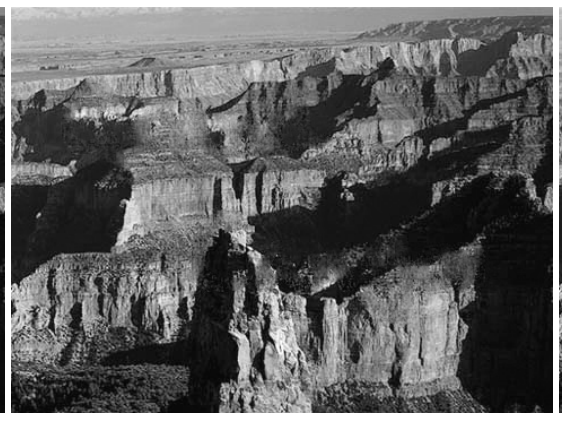

(b)

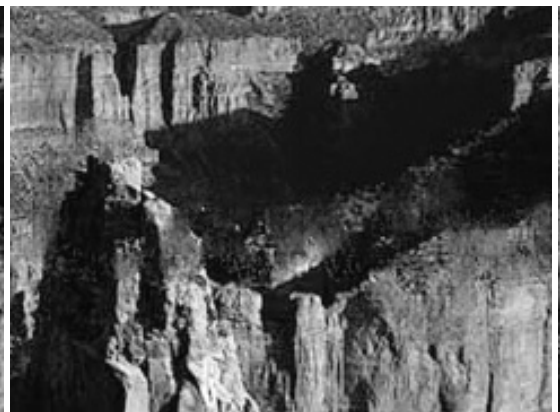

(e)

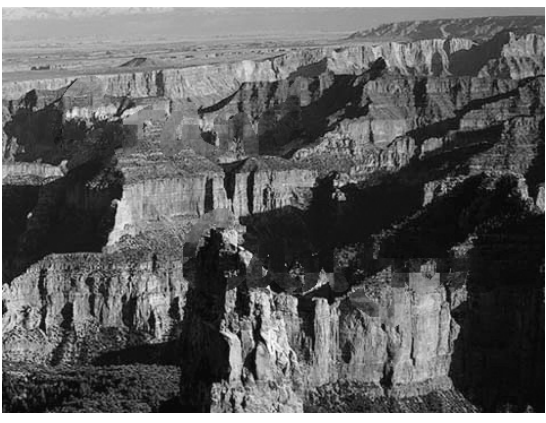

(c)

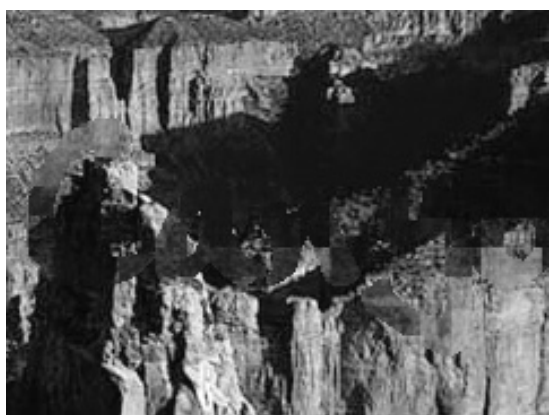

(f)

Fig. 6 (a) Corrupted image including text regions, (b) Image reconstructed by the proposed method $(28.13 \mathrm{~dB})$, (c) Image reconstructed by the traditional method $(25.82 \mathrm{~dB})$, (d) Zoomed portion of the original image, (e) Zoomed portion of (b), (f) Zoomed portion of (c). 


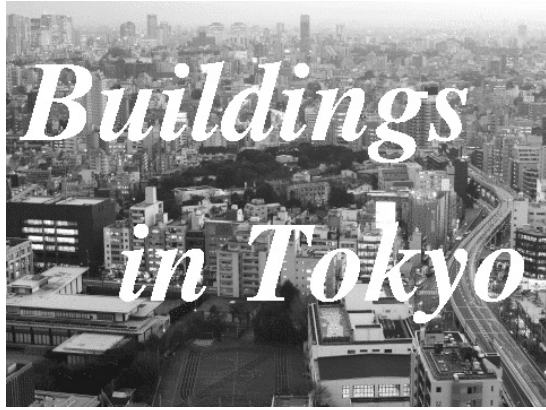

(a)

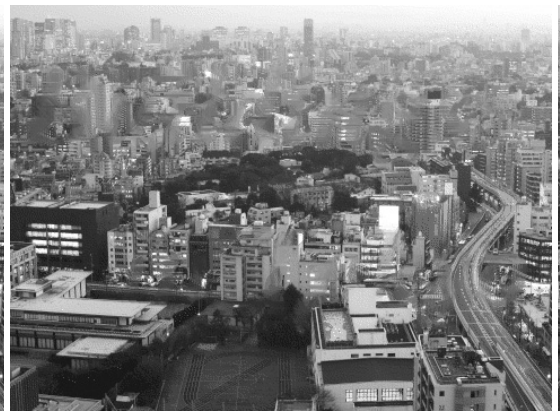

(b)

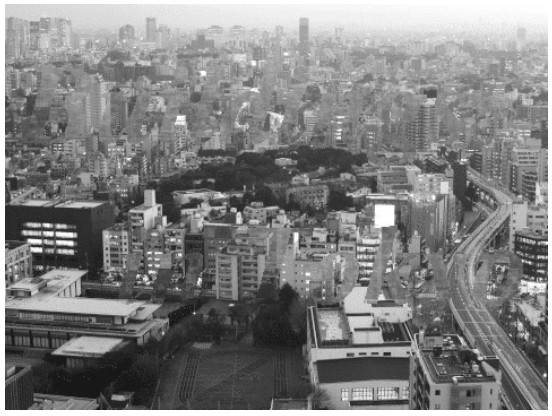

(c)

Fig. 8 (a) Target image including the text "Buildings in Tokyo," (b) Image reconstructed by the proposed method $(27.48 \mathrm{~dB})$, (c) Image reconstructed by the traditional method $(25.91 \mathrm{~dB})$.

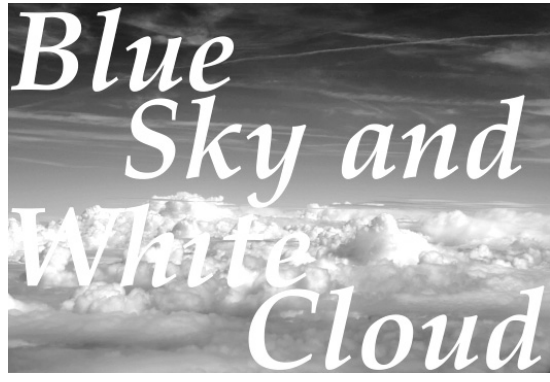

(a)

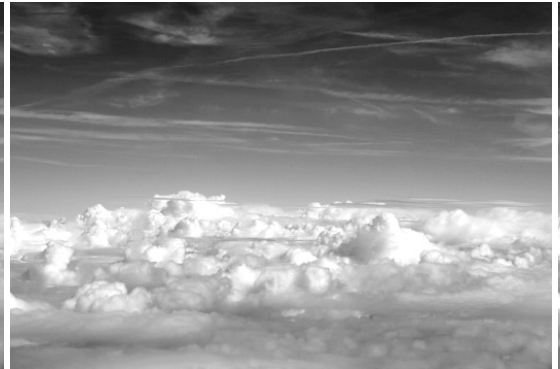

(b)

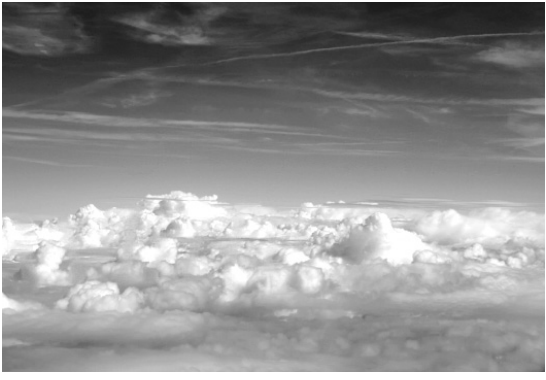

(c)

Fig. 9 (a) Target image including the text "Blue Sky and White Cloud," (b) Image reconstructed by the proposed method $(37.33 \mathrm{~dB})$, (c) Image reconstructed by the traditional method $(31.83 \mathrm{~dB})$.

experimental results are shown in Figs. 8 and 9. Compared to the results obtained by using the conventional method, it can be seen that various kinds of textures can be accurately restored by using the proposed method.

In order to quantitatively evaluate the performance of the proposed method, we use three texture images [15] and performed the same simulations as those for which results are shown in Fig. 6. Table 1 shows the PSNR ${ }^{\dagger}$ of the results of reconstruction. This table also includes the results shown in Figs. 6-9. Further, Table 2 shows the number of clipped local images including missing areas and that of clipped local images not including missing areas for the six test images in Table 1 . It can be seen that our method has achieved an improvement of $0.8-5.5 \mathrm{~dB}$ over the conventional method. Therefore, good performance of the proposed method was verified by the experiments.

Finally, we verify the computational complexity of the proposed method. Figure 10 shows the converged errors versus the iteration number in our POCS algorithm. From this figure, the errors are converged in the first few iteration. Since the POCS algorithm guarantees strong convergence [9], we can obtain the reconstruction results with few low computational complexity. Further, we show computation time of the proposed method and the conventional method in Table 3. This simulation is performed on a personal computer using AMD Athlon(tm) 64 X2 Dual Core Processor $3800+2.0 \mathrm{GHz}$ CPU with 1.0 Gbytes RAM. Both
Table 1 Performance comparison (PSNR) of the proposed method and the conventional method.

\begin{tabular}{c|c|c}
\hline Image & Conventional method & Proposed method \\
\hline \hline Flowers.0001 & $25.48 \mathrm{~dB}$ & $\mathbf{2 6 . 2 5 ~ d B}$ \\
\hline Bark.0009 & $23.87 \mathrm{~dB}$ & $\mathbf{2 4 . 9 7 ~ d B}$ \\
\hline Brick.0001 & $35.44 \mathrm{~dB}$ & $\mathbf{3 7 . 6 9 d B}$ \\
\hline Fig. 6 & $25.82 \mathrm{~dB}$ & $\mathbf{2 8 . 1 3 ~ d B}$ \\
\hline Fig. 8 & $25.91 \mathrm{~dB}$ & $\mathbf{2 7 . 4 8 ~ d B}$ \\
\hline Fig. 9 & $31.83 \mathrm{~dB}$ & $\mathbf{3 7 . 3 3 ~ d B}$
\end{tabular}

the proposed method and the conventional method are implemented by using Matlab. As shown in this table, we can see the proposed method realizes lower computational cost than that of the conventional method. Therefore, several applications of the proposed method can be expected.

\section{Conclusions}

In this paper, we have proposed a new POCS-based framework for reconstruction of missing textures in still images. The proposed method performs a texture classification for local images within the target image and introduces the nonlinear eigenspace of each cluster into the constraint of the

${ }^{\dagger} \mathrm{PSNR}=10 \log _{10} \frac{\mathrm{MAX} 2}{\mathrm{MSE}}$, where MAX denotes the maximum value of intensities and MSE is the mean square error between the original image and the reconstructed image. 
Table 2 The number of local images including missing areas and that of local images not including missing areas.

\begin{tabular}{c|c|c}
\hline Test image & $\begin{array}{c}\text { Number of local images } \\
\text { including missing areas }\end{array}$ & $\begin{array}{c}\text { Number of local images } \\
\text { not including missing areas }(=N)\end{array}$ \\
\hline \hline Flowers.0001 & 586 & 854 \\
\hline Bark.0009 & 586 & 854 \\
\hline Brick.0001 & 586 & 854 \\
\hline Fig. 6 & 586 & 854 \\
\hline Fig. 8 & 823 & 617 \\
\hline Fig.9 & 1037 & 531 \\
\hline
\end{tabular}

Table 3 Comparison of computational time (sec) between the proposed method and the conventional method. Missing rate represents the rate of missing pixels in the target image.

\begin{tabular}{c|c|c|c|c|c}
\hline Test image & Image size & Bit level & Missing rate & Conventional method & Proposed method \\
\hline \hline Flowers.0001 & $480 \times 359$ & 8-bit gray & 0.089 & $4.44 \times 10^{3}$ & $2.57 \times 10^{2}$ \\
\hline Bark.0009 & $480 \times 359$ & 8-bit gray & 0.089 & $4.35 \times 10^{3}$ & $2.62 \times 10^{2}$ \\
\hline Brick.0001 & $480 \times 359$ & 8-bit gray & 0.089 & $4.30 \times 10^{3}$ & $2.72 \times 10^{2}$ \\
\hline Fig. 6 & $480 \times 359$ & 24-bit color & 0.089 & $1.01 \times 10^{4}$ & $6.13 \times 10^{2}$ \\
\hline Fig. 8 & $480 \times 359$ & 24-bit color & 0.11 & $1.00 \times 10^{4}$ & $5.69 \times 10^{2}$ \\
\hline Fig.9 & $527 \times 359$ & 24-bit color & 0.13 & $9.97 \times 10^{3}$ & $6.16 \times 10^{2}$ \\
\hline
\end{tabular}

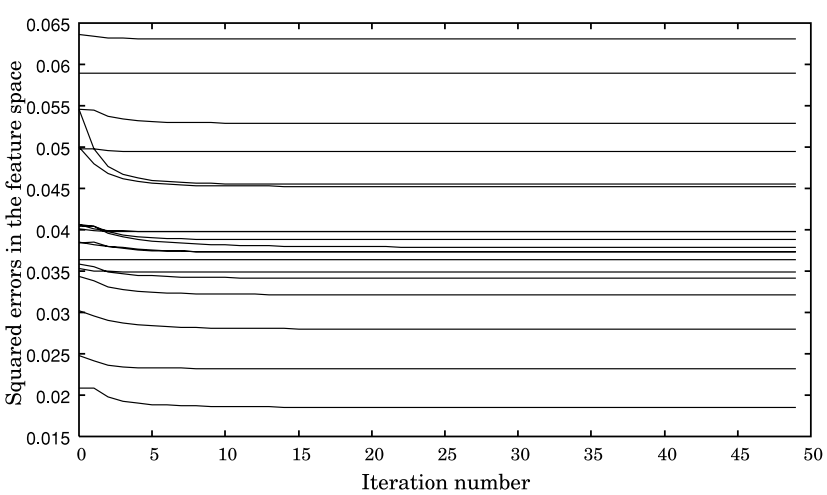

Fig. 10 Errors converged by our POCS algorithm versus iteration number in the experiment of Fig. 6 .

POCS-based reconstruction algorithm. This approach provides a better ground for improving the capability of approximating the same kinds of textures within the target image. Furthermore, by monitoring the errors converged by the POCS algorithm, selection of the same kinds of textures for the target local image including missing intensities can be achieved. The above two novel approaches enable adaptive restoration of the missing textures, and the proposed method therefore has good performance. Our experimental results verified the superiority of our POCS-based reconstruction technique. Impressive improvements in both objective and subjective measures have been achieved.

In this study, we manually set parameters such as the size of local images and the number of clusters. It is desirable that these values can be determined from the observed images adaptively. Thus, we need to complement this determination algorithm. Extension of the framework to texture reconstruction of other types of missing imagery data is also needed for various applications. Last, we would like to study these ideas for interpolation in video data. These topics will be the subject of subsequent reports.

\section{Acknowledgment}

This work was supported under project SCOPE (Strategic Information and Communications R\&D Promotion Programme) of Japanese Ministry of Internal Affairs and Communications.

\section{References}

[1] C. Ballester, M. Bertalmio, V. Caselles, and G. Sapiro, "Filling-in by joint interpolation of vector fields and gray levels," IEEE Trans. Image Process., vol.10, no.8, pp.1200-1211, 2001.

[2] T.F. Chan and J. Shen, "Nontexture inpainting by curvature-driven diffusions," J. Visual Communication and Image Representation, vol.12, no.4, pp.436-449, 2001.

[3] A. Rares, M.J.T. Reinders, and J. Biemond, "Edge-based image restoration," IEEE Trans. Image Process., vol.14, no.10, pp.1454 1468, 2005.

[4] A.A. Efros and T.K. Leung, "Texture synthesis by nonparametric sampling," IEEE Int. Conf. Computer Vision, pp.1033-1038, Corfu, Greece, Sept. 1999.

[5] A. Kokaram, "A statistical framework for picture reconstruction using 2D AR models," Image Vis. Comput., vol.22, no.2, pp.165-171, Feb. 2004.

[6] B. Schölkopf, S. Mika, C.J.C Burges, P. Knirsch, K.-R. Müller, G. Rätsch, and A.J. Smola, "Input space versus feature space in kernelbased methods," IEEE Trans. Neural Netw., vol.10, no.5, pp.10001017, 1999.

[7] S. Mika, B. Schölkoph, A. Smola, K.-R. Müller, M. Scholz, and G. Rätsch, "Kernel PCA and de-noising in feature spaces," Advances in Neural Information Processing Systems 11, pp.536-542, 1999.

[8] A. Hirani and T. Totsuka, "Combining frequency and spatial domain information for fast interactive image noise removal," Computer Graphics, SIGRAPH 96, pp.269-276, 1996.

[9] D.C. Youla and H. Webb, "Image restoration by the method of convex projections: Part 1-Theory,” IEEE Trans. Med. Imaging, vol.MI-1, no.2, pp.81-94, Oct. 1982.

[10] A. Zakhor, "Iterative procedures for reduction of blocking effects in transform image coding," IEEE Trans. Circuits Syst. Video Technol., vol.2, no.1, pp.91-95, March 1992. 
[11] J. Mercer, "Functions of positive and negative type and their connection with the theory of integral equations," Trans. Lond. Phil. Soc. (A), vol.209, pp.415-446, 1909.

[12] J.T.Y. Kwok and I.W.H. Tsang, "The pre-image problem in kernel methods," IEEE Trans. Neural Netw., vol.15, no.6, pp.1517-1525, 2004

[13] K.I. Kim, M.O. Franz, and B. Schölkoph, "Iterative kernel principal component analysis for image modeling," IEEE Trans. Pattern Anal. Mach. Itell., vol.27, no.9, pp.1351-1366, 2005.

[14] T. Hastie, R. Tibshirani, and J. Friedman, The element of statistical learning. Data mining, inference and prediction, Springer, 2001

[15] P. Brodatz, Textures: A Photographic Album for Artists and Designers, Dover Publications, New York, 1966.

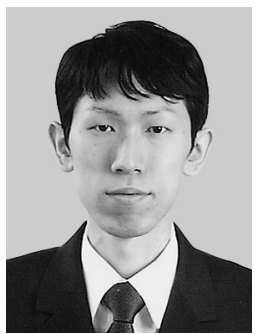

Takahiro Ogawa received his B.S. and M.S. degrees in Electronics and Information Engineering from Hokkaido University, Japan in 2003 and 2005, respectively. He is currently a Ph.D. student of the Graduate School of Information Science and Technology, Hokkaido University. His research interests are in image restoration and its applications.

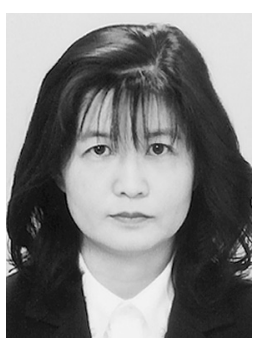

Miki Haseyama received her B.S., M.S. and Ph.D. degrees in Electronics from Hokkaido University, Japan in 1986, 1988 and 1993, respectively. She is currently a professor in the Graduate School of Information Science and Technology, Hokkaido University. Her research interests are in digital signal processing and its applications. 